\title{
Contribuição do Planejamento Orçamentário no Gerenciamento do Laboratório de Hemodinâmica: Simulação Aplicada à Gestão dos Serviços de Hemodinâmica
}

\author{
Carlos Roberto Cardoso' ${ }^{1}$ Elvis Preslli Prestes ${ }^{1}$, Cristiano de Oliveira Cardoso ${ }^{1}$, Rolando Beulke ${ }^{2}$
}

\section{RESUMO}

Introdução: $O$ instrumento necessário de gestão para qualquer empresa é o emprego de um orçamento planejado, que permitirá calcular e estimar custos, para melhor aplicação dos recursos. A utilização desse recurso gerencial no setor de hemodinâmica tem a finalidade de compatibilizar o aspecto financeiro, sem prejuízo do resultado médico. O objetivo do presente estudo é apresentar a contribuição do planejamento orçamentário no gerenciamento do laboratório de hemodinâmica. Método: Por meio de uma simulação, foi realizado um cálculo orçamentário em um laboratório de hemodinâmica fictício com capacidade de realização de 160 procedimentos/mês $(75 \%$ de cateterismos diagnósticos e $25 \%$ de angioplastias). Foram elaborados três cenários de produção acordados com a capacidade de realização de exames do serviço: pessimista $(60 \%$ da capacidade total), moderado $(75 \%)$ e otimista $(95 \%)$. O orçamento foi executado de acordo com as seguintes peças orçamentárias: orçamento de vendas, orçamento de produção, orçamento de despesas operacionais e demonstrativo de resultados do exercício. Resultados: De acordo com a simulação, estimou-se um acréscimo na receita bruta de $25,7 \%$ no cenário moderado e de $59,2 \%$ no otimista à medida que a capacidade total de produção é alcançada. Os custos diretos representam cerca de $58 \%$ do total da receita líquida. A rentabilidade foi de $7,9 \%$ no cenário pessimista, de $14,87 \%$ no moderado e de $20,33 \%$ no otimista. Conclusão: O planejamento orçamentário é uma ferramenta administrativa que pode ser aplicada à gestão dos laboratórios de hemodinâmica, auxiliando na racionalização de gastos, custos, despesas e desperdícios existentes.

DESCRITORES: Gestão em saúde. Planejamento. Orçamento. Hemodinâmica.

\section{ABSTRACT}

Contribution of Budget Planning to Catheterization Laboratory Management: A Simulation Applied to the Administration of Hemodynamic Services

Background: Budget planning is a tool required to manage any company. It enables cost calculation and estimates to make the best use of the available resources. The use of this management tool in the catheterization laboratory is aimed at establishing a balance between financial aspects without interfering with the medical outcome. The objective of this study is to report the contribution of a planned budget in the catheterization laboratory management. Method: Using a simulation study, a fictitious budget calculation was created to manage a cathlab with a total production capacity of 160 procedures/month (75\% of diagnostic procedures and $25 \%$ of intervention procedures). Three hypothetic scenarios were developed according to the production capacity: pessimistic $(60 \%$ of the overall production capacity), moderate $(75 \%)$ and optimistic (95\%). The budget was prepared according to the following estimates: sales estimate, production estimate, operational expenses estimate and financial statement for the period. Results: According to the simulation, there was a raw income increase of $25.7 \%$ for the moderate scenario and $59.2 \%$ for the optimistic scenario as the total production capacity is achieved. Direct costs represent around $58 \%$ of the net income. Profitability was $7.9 \%$ in the pessimistic scenario, $14.87 \%$ in the moderate scenario and $20.33 \%$ in the optimistic scenario. Conclusion: Budget planning is an administrative tool which may be applied to cathlab management, helping reduce costs, expenses and waste.

KEY-WORDS: Health management. Budget. Management. Hemodynamics.

\footnotetext{
1 Instituto de Cardiologia do Rio Grande do Sul - Fundação Universitária de Cardiologia (IC-FUC) - Porto Alegre, RS, Brasil.

2 Universidade Federal do Rio Grande do Sul (UFRGS) - Porto Alegre, RS, Brasil.

Correspondência: Carlos Roberto Cardoso. Instituto de Cardiologia do Rio Grande do Sul. Av. Princesa Isabel, 395 - Porto Alegre, RS, Brasil - CEP 90620-001

E-mail: carlosrcardoso@terra.com.br

Recebido em: 20/12/2009 • Aceito em: 25/2/2010
} 
A consolidação da medicina baseada em evidências tornou as condutas médicas mais críticas e menos dependentes da experiência pessoal, ${ }^{1,2}$ estimulando o emprego de diretrizes na assistência médica, com o objetivo de melhorar os resultados para os pacientes. No entanto, a principal limitação da medicina baseada em evidências é ser uma ferramenta inefetiva na redução dos custos com a promoção da saúde. ${ }^{3}$

$\mathrm{Na}$ assistência à saúde os melhores prestadores de serviço devem ser os mais eficientes e não, necessariamente, os mais dispendiosos. ${ }^{3}$ A boa qualidade na promoção da saúde é caracterizada por ser menos onerosa, pois elabora diagnósticos precisos, evita tratamentos ineficazes, reduz taxas de complicações e permite menor tempo de internação. ${ }^{3,4}$

A medicina baseada em evidências permite analisar certos aspectos monetários (custo-benefício) e o impacto social (resultado nos desfechos) de determinado tratamento ou método diagnóstico; ${ }^{1}$ entretanto, aspectos específicos de gerenciamento e planejamento orçamentário não são contemplados.

O instrumento necessário de gestão para qualquer empresa é o emprego de um orçamento planejado, independentemente do porte ou da atividade, e que permitirá calcular e estimar custos, para meIhor aplicação dos recursos. Orçamento é a ferramenta de controle por excelência de todo o processo operacional de uma empresa, pois envolve todos os seus setores. ${ }^{5,6}$ Para compatibilizar sucesso médico com recursos disponíveis é necessário um planejamento adequado, que possibilite perceber a realidade, avaliar a trajetória, construir um referencial futuro, estruturar e reavaliar os processos utilizados na assistência à saúde. ${ }^{7}$

É objetivo do presente estudo apresentar a contribuição do planejamento orçamentário no gerenciamento do laboratório de hemodinâmica.

\section{METÓDO}

\section{Objeto de estudo}

O presente estudo teve como base a análise e projeção dos dados referentes a uma simulação de gerenciamento de um laboratório de hemodinâmica. Partiu-se do pressuposto de que um laboratório de hemodinâmica poderia executar cerca de 160 procedimentos/mês. Em cima dessa premissa, foi ficticiamente elaborado um planejamento orçamentário.

\section{Características do laboratório de hemodinâmica}

Nessa simulação foi considerado um laboratório de hemodinâmica com apenas uma máquina para realização de procedimentos. São parte integrante do quadro de funcionários uma enfermeira, quatro técnicos de enfermagem, duas auxiliares de higienização e três secretárias. Foi considerando um horário de funcionamento de oito horas diárias e com a realização de um procedimento por hora, totalizando oito procedimentos/dia em 20 dias úteis. Tanto a esterilização de materiais quanto a recuperação pós-procedimento são realizadas nas dependências do hospital, não sendo gerados custos ao laboratório relacionados a esses serviços. Não foi considerado reuso de cateteres, balões, guias e introdutores.

\section{Elaboração do orçamento}

Para projetar o orçamento simulado foram consideradas, fundamentalmente, duas fontes referentes a procedimentos hemodinâmicos: Sociedade Latino-Americana de Cardiologia Intervencionista (SOLACI) e dados internos do Instituto de Cardiologia do Rio Grande do Sul - Fundação Universitária de Cardiologia (IC-FUC). No registro da $\mathrm{SOLACl}^{8}$ de 1998 a 2005, foram realizados 1.681.584 procedimentos: $74,9 \%$ diagnósticos e $25,1 \%$ terapêuticos. Dados internos do IC-FUC revelam que no ano de 2008 cerca de $28 \%$ dos procedimentos hemodinâmicos foram do tipo angioplastia. Com base nesses dados reais, nossa simulação considerou uma produção de 160 procedimentos/mês, totalizando $25 \%$ de angioplastias e $75 \%$ de cateterismos diagnósticos. Foi considerada, para efeito de cálculo, angioplastia com implante de apenas um stent.

\section{Detalhamento do orçamento}

A formatação do planejamento orçamentário foi simulada contendo as seguintes peças orçamentárias:

- orçamento de vendas;

- orçamento de produção;

- orçamento de despesas operacionais;

- demonstrativo de resultados do exercício.

Seus componentes são, sumariamente, descritos a seguir:

1. Orçamento de vendas: composto pelo número de procedimentos realizados em um semestre, receita bruta, glosas e receita líquida. As glosas foram estimadas em $0,5 \%$ da receita bruta em cada cenário simulado.

2. Orçamento de produção: tem origem no cálculo antecipado dos procedimentos a serem vendidos. Na elaboração do orçamento de produção devem ser considerados os custos dos insumos e os custos dos honorários médicos. Os insumos seriam compostos por medicamentos, OPME (órteses, próteses e materiais especiais) e outros materiais (material de enfermagem, material médico-hospitalar, gases medicinais, gêneros alimentícios e material radiológico). A soma dos insumos e da mão de obra direta forma os custos diretos (variáveis).

3. Orçamento de despesas operacionais: é constituído por materiais secundários, despesas com pessoal 
não-médico; consumo de energia elétrica, água, telefone, etc.; depreciações de máquinas e equipamentos; serviços de terceiros e manutenção.

4. O demonstrativo de resultados do exercício para o período considerado é elaborado a partir dos elementos fornecidos pelos orçamentos de vendas, de produção e de despesas operacionais.

\section{Cenários das simulações}

Seguindo os princípios de contabilidade gerencial, é adequado elaborar três cenários para expressar o orçamento: um pessimista, um moderado e um otimista. Para criar os três cenários foi considerada a capacidade total de produção do laboratório de hemodinâmica (160 procedimentos/mês). No cenário pessimista utilizaram-se $60 \%$ da capacidade do laboratório de hemodinâmica (capacidade total de produção); no moderado, 75\% da capacidade total de produção; e no cenário otimista, $95 \%$ da capacidade total de produção. A simulação orçamentária considerou um período de seis meses.

\section{Apresentação dos dados financeiros}

O orçamento projetado é apresentado em valores físicos e monetários. As unidades físicas são expressas em números absolutos, médias e porcentagens. As unidades monetárias são expressas no valor da moeda nacional (real - R\$).

A correção dos valores orçamentários, quando necessária, foi realizada por meio de indicadores oficiais, sendo os principais descritos a seguir: dólar (materiais importados - valor de câmbio em que um dólar equivale a 1,79 real), inflação (fundamentalmente salários - valor de referência igual a 4,7\%) e índice nacional de preços ao consumidor (INPC - valor de referência igual a 6,06\%). Utilizou-se, para fins de orçamento, somente uma fonte pagadora. Os valores de referência foram os mesmos atualmente reembolsados pelo Sistema Único de Saúde ${ }^{9}$ (SUS), referentes aos procedimentos de cateterismo cardíaco ( $R \$ 614,72)$ e angioplastia ( R\$ 3.075,48).

Sendo um orçamento departamental (o laboratório é um departamento dentro de um hospital), não foram realizados orçamentos que transcendem o departamento, ou seja, orçamento de caixa, orçamento fiscal, orçamento de investimentos e impostos devidos. A simulação considerou, também, que o laboratório de hemodinâmica faz parte de um hospital filantrópico, logo não se aplicam, na simulação, impostos devidos. Os encargos sociais, obrigação legal de qualquer empregador, estão contemplados no custo dos salários. Ao salário base foram adicionados 35\% para essa finalidade.

\section{Definições}

No estudo serão utilizados alguns termos técnicos que são definidos a seguir:
- período base: representa o período fundamental de custos do laboratório (na simulação os custos do cenário pessimista são iguais aos do período base);

- receita bruta: número de procedimentos multiplicado pelo valor de cada procedimento;

- receita líquida: obtida pela subtração da receita bruta pelas glosas;

- margem de contribuição: receita líquida, subtraídos os custos diretos;

- resultado operacional: é o resultado da subtração da margem de contribuição pelos custos diretos;

- resultado gerencial: resultado operacional acrescido ao resultado não-operacional;

- rentabilidade: resultado gerencial dividido pela receita líquida;

- resultado do exercício: resultado operacional, subtraídas as provisões.

\section{RESULTADOS}

Em nossa simulação a capacidade total de produção foi de 160 procedimentos/mês, constituída por 40 angioplastias (25\%) e 120 cateterismos diagnósticos (75\%). No cenário pessimista $(60 \%$ da capacidade total de produção) seriam realizados 576 procedimentos; no moderado $(75 \%$ da capacidade total de produção), 720 ; e no otimista (95\% da capacidade total de produção), 920 exames.

Na Tabela 1 são apresentados os valores simulados com os gastos do orçamento de vendas.

O orçamento de produção é detalhado na Tabela 2. Observa-se que, pela alta rentabilidade do laboratório de hemodinâmica, a receita bruta aumenta significativamente nos diferentes cenários de projeção.

Dados das despesas operacionais estão apontados na Tabela 3. Nota-se a ocorrência de pequeno incremento em seus valores, mantendo-se praticamente fixas essas despesas no período projetado de seis meses.

$\mathrm{Na}$ Tabela 4 é apresentado o demonstrativo de resultados do exercício no período projetado.

\section{DISCUSSÃO}

Neste trabalho procuramos identificar a aplicabilidade do planejamento orçamentário no departamento de hemodinâmica.

Entre as funções básicas da administração duas relacionam-se diretamente ao processo de elaboração do sistema orçamentário: planejamento e controle de resultados..$^{5,6,10}$ O planejamento em curto prazo seria compreendido de seis meses a um ano, com a finalidade de se obter o melhor emprego dos recursos existentes, tanto físicos como monetários. ${ }^{10}$ 
TABELA 1

Descrição dos componentes do orçamento de vendas e suas projeções nos cenários pessimista, moderado e otimista

\begin{tabular}{lcccc}
\hline & & \multicolumn{3}{c}{ Cenário projetado } \\
\cline { 3 - 5 } Orçamento de vendas & Período base & Pessimista & Moderado & Otimista \\
\hline Número de procedimentos & 576 & 576 & 720 & 912 \\
Receita bruta $(\mathrm{R} \$)$ & $708.428,18$ & $708.428,18$ & $885.535,20$ & $1.121 .677,90$ \\
Glosas* $(\mathrm{R} \$)$ & $3.542,14$ & $3.541,80$ & $4.427,24$ & $5.608,39$ \\
Receita líquida $(\mathrm{R} \$)$ & $704.886,04$ & $704.886,38$ & $881.107,96$ & $1.116 .069,51$ \\
\hline * $0,5 \%$ da receita bruta. & & & & \\
\hline
\end{tabular}

TABELA 2

Descrição dos componentes do orçamento de produção e suas projeções nos cenários pessimista, moderado e otimista

\begin{tabular}{lcccc}
\hline & & \multicolumn{3}{c}{ Cenário projetado } \\
\cline { 3 - 5 } Orçamento de produção* & Período base & Pessimista & Moderado & Otimista \\
\hline Medicamentos & $20.856,13$ & $20.856,13$ & $25.902,03$ & $32.857,09$ \\
OPME & $250.273,51$ & $250.273,51$ & $310.824,41$ & $394.285,04$ \\
Material de enfermagem & $6.673,96$ & $6.673,96$ & $8.288,65$ & $10.514,27$ \\
Outros materiais & 500,55 & 500,55 & 621,65 & 788,57 \\
Honorários médicos & $138.818,37$ & $138.818,37$ & $172.403,94$ & $218.696,77$ \\
Custos diretos & $417.122,51$ & $417.122,51$ & $518.040,68$ & $657.141,73$ \\
Receita líquida & $704.886,04$ & $704.886,04$ & $881.107,96$ & $1.116 .069,51$ \\
Margem de contribuição & $287.763,53$ & $287.763,53$ & $363.067,28$ & $458.927,78$ \\
\hline * Valores em reais. & & & \\
OPME $=$ órteses, próteses e materiais especiais. & & & \\
\hline
\end{tabular}

TABELA 3

Descrição dos componentes do orçamento de despesas operacionais

\begin{tabular}{lcc}
\hline Orçamento de despesas operacionais* & Período base & Período projetado \\
\hline Despesas com pessoal & $136.097,70$ & $146.420,83$ \\
Custos gerais e com materiais & $37.168,53$ & $39.621,65$ \\
Provisões & $44.195,37$ & $47.112,26$ \\
Despesas gerais (administrativas) & $81.363,90$ & $86.733,91$ \\
Despesas (custos) fixas & $217.461,60$ & $233.154,74$ \\
\hline * Valores em reais. & & \\
\hline
\end{tabular}

Para planejar é preciso conhecer intimamente a situação atual do sistema e definir aquela que se pretende atingir, sendo para isso necessário tomar decisões que afetam a estrutura, os processos de produção e o produto do sistema. ${ }^{11}$ Planejar é uma estratégia para aumentar as chances de sucesso de uma empresa num mundo de negócios que muda constantemente.
Orçar significa processar todos os dados constantes do sistema de informações contábeis atuais e introduzir os dados previstos para o próximo exercício, considerando as alterações já definidas para o próximo exercício. ${ }^{5} \mathrm{O}$ orçamento deve apresentar flexibilidade em sua aplicação, permitindo corrigir desvios ocorridos. No setor privado, o orçamento deve ade- 
Cardoso CR, et al. Contribuição do Planejamento Orçamentário no Gerenciamento do Laboratório de Hemodinâmica: Simulação Aplicada à Gestão dos Serviços de Hemodinâmica. Rev Bras Cardiol Invasiva. 2010;18(1):62-7.

TABELA 4

Demonstrativo de resultados do exercício

\begin{tabular}{lcccc}
\hline & & \multicolumn{3}{c}{ Cenário projetado } \\
\cline { 3 - 5 } & Período base & Pessimista & Moderado & Período base \\
\hline Resultado do exercício (R\$) & $26.106,56$ & $7.496,57$ & $82.800,32$ & $178.660,82$ \\
Produção (procedimentos) & 576 & 576 & 720 & 912 \\
Renda líquida (R\$) & $704.886,04$ & $704.886,38$ & $881.107,96$ & $1.116 .069,51$ \\
Custos diretos (R\$) & $417.122,51$ & $417.122,51$ & $518.040,68$ & $657.141,73$ \\
Despesas operacionais (R\$) & $217.461,60$ & $233.154,70$ & $233.154,70$ & $233.154,70$ \\
Lucro operacional (R\$) & $70.301,93$ & $54.608,83$ & $129.912,58$ & $225.773,08$ \\
Resultado não-operacional (R\$) & $1.076,00$ & $1.076,00$ & $1.076,00$ & $1.076,00$ \\
Margem de contribuição (R\$) & $287.763,53$ & $287.763,53$ & $363.067,28$ & $458.927,78$ \\
Resultado gerencial (R\$) & $71.377,93$ & $55.684,83$ & $130.988,58$ & $226.849,08$ \\
Rentabilidade (\%) & 10,13 & 7,90 & 14,87 & 20,33 \\
\hline
\end{tabular}

quar a despesa à receita; no setor público, o orçamento deve adequar a receita à despesa..$^{5,6}$

O orçamento deve apresentar algumas características $^{6,10}$ de projeção para o futuro, flexibilidade na aplicação, e participação direta dos responsáveis, devendo também envolver todas as atividades operacionais do departamento, ser prático com princípios e padrões uniformes, revelar economicidade, e ser expresso em valores físicos e monetário, os quais devem ser definidos previamente.

O orçamento de vendas é o marco inicial do planejamento orçamentário. Ele auxilia os gestores da instituição a formular o preço de venda do produto (procedimentos) e negociar com os tomadores de serviços. ${ }^{6}$ Em sua elaboração devem ser observados alguns aspectos especificamente relacionados às características do departamento: potencial do mercado e nível de demanda. O orçamento de vendas deve ser realista e flexível. 5,6,10 No orçamento de vendas devese responder à questão: o que vender? No caso da hemodinâmica, vendem-se procedimentos. No serviço de hemodinâmica as quantidades vendida e produzida são as mesmas, pois não é possível estocar procedimentos. O orçamento de vendas auxilia para projetar metas.

No orçamento simulado do estudo verifica-se que para atingir um cenário moderado seria necessário aumentar a produção (procedimentos) em 25\% em relação ao período base, o que equivaleria a aumentar 24 procedimentos por mês. Para atingir o cenário otimista deveria haver aumento de cerca de $58 \%$ na produção, o mesmo que aumentar 56 procedimentos por mês.

Na análise do OV algumas questões podem surgir: "as metas são viáveis de ser alcançadas?", "há demanda para o serviço?". Várias outras questões de importância para o desempenho do serviço também podem aparecer.
O orçamento de produção tem origem no cálculo antecipado dos procedimentos a serem vendidos e não tem sentido uma produção ser projetada se não houver mercado para o produto, pois a receita auferida, além de cobrir os custos operacionais e não-operacionais, deverá proporcionar uma sobra ${ }^{10}$. A elaboração do orçamento de produção é documento básico para avaliar compras e consumo de materiais especiais, assim como o custo da mão de obra direta. Em alguns serviços o custo da mão de obra direta (representada pelos intervencionistas) é pago pelos convênios com repasse por intermédio do hospital. O orçamento de produção expressa a quantidade de procedimentos (produção) realizados durante o período orçamentário com seu respectivo custo, estabiliza a produção e racionaliza os custos. ${ }^{5,6}$ Fato importante a ser considerado no orçamento de produção do SH é que o serviço prestado não pode ser estocado. A soma dos insumos e da mão de obra direta forma os custos diretos. No orçamento de produção os custos diretos, também chamados de custos variáveis, expressam os custos dos procedimentos e auxiliam na redução dos desperdícios de materiais. A margem de contribuição impacta diretamente no resultado operacional do departamento.

Na simulação atual o cenário mais provável de atingir seria o moderado. Pode-se verificar que as OPME são responsáveis por $60 \%$ dos custos diretos. Isso deve influenciar o grau de negociação do gestor com os fornecedores desses materiais. A margem de contribuição aumentará em 26,2\%.

O orçamento de despesas operacionais será constituído por despesas administrativas (gerais), vendas (comerciais), financeiras e tributárias, ou seja, por todos os gastos que incorrerão no período projetado, exceto os custos de produção. ${ }^{6}$ Dentre as principais despesas operacionais encontram-se: a) materiais secundários; b) despesas com pessoal não-médico; c) consumo de energia 
Cardoso CR, et al. Contribuição do Planejamento Orçamentário no Gerenciamento do Laboratório de Hemodinâmica: Simulação Aplicada à Gestão dos Serviços de Hemodinâmica. Rev Bras Cardiol Invasiva. 2010;18(1):62-7.

elétrica, água, telefone, etc.; d) depreciações de máquinas e equipamentos; e) serviços de terceiros e manutenção. No departamento de hemodinâmica as despesas operacionais compreendem custos com pessoal de enfermagem e técnicos, com pessoal burocrático, com materiais e depreciação dos equipamentos. Trata-se de gastos não ligados às operações de produção e vendas do departamento, embora indispensáveis à manutenção do serviço. No departamento de hemodinâmica nos fixamos somente nas despesas administrativas, pois vendas e questões tributárias e financeiras são da alçada da administração central.

A gestão adequada do orçamento de despesas operacionais se reflete diretamente no resultado operacional (lucro operacional). Nos períodos de dificuldades são as despesas operacionais que inicialmente devem ser reduzidas. Um item importante que os responsáveis pelos departamentos de hemodinâmica deveriam considerar é o que se refere às provisões. Um porcentual do valor das provisões deveria ser destinado diretamente para o departamento, com o objetivo de permitir atualizações no laboratório. No modelo atual simulado o valor projetado seria de $\mathrm{R} \$ 47.112,26$ no semestre.

Após elaboração dos orçamentos de vendas, de produção e de despesas operacionais é exposto o demonstrativo de resultados para o exercício projetado, que permite avaliar sinteticamente o planejamento realizado. ${ }^{5,6,10,12,13}$ A principal característica do demonstrativo de resultados do exercício será confrontar os valores realizados e estimados para verificar a eficiência e a eficácia do plano geral. O demonstrativo de resultados do exercício para o período considerado é elaborado a partir dos elementos fornecidos pelos orçamentos de vendas, produção e despesas operacionais. Esse instrumento permite avaliar o desempenho do setor e auxilia no alcance dos objetivos e metas traçados. Isso favorece a criação de um sistema de avaliação que tende a verificar a eficiência do setor.

O demonstrativo de resultados do exercício do departamento de hemodinâmica auxilia a administração da instituição a elaborar seu planejamento estratégico.

\section{LIMITAÇÕES DO ESTUDO}

Em virtude da singularidade do estudo, a discussão fica prejudicada, pois desconhecemos trabalho que utilize o planejamento orçamentário especificamente na área da hemodinâmica. Portanto, não encontramos critérios para compará-lo com outros estudos do gênero. Outras questões pertinentes, como a carga tributária, não foram consideradas nessa simulação, pois o laboratório em questão faz parte de um hospital filantrópico. Por tratar-se de uma simulação em que o objeto de estudo é um departamento dentro de um hospital filantrópico, nossa simulação não pode ser extrapolada integralmente para laboratórios de hemodinâmica totalmente terceirizados.

\section{CONCLUSÃO}

Este trabalho evidencia a possibilidade do emprego do planejamento orçamentário no departamento de hemodinâmica. Auxilia na racionalização de gastos, custos, despesas e desperdícios existentes no departamento. Permite, também, à administração da instituição planejar o futuro e instituir metas a serem alcançadas.

\section{VINCULAÇÃO ACADÊMICA}

O presente estudo foi apresentado pelo hemodinamicista Carlos Roberto Cardoso para obtenção do título de Especialista em Gestão em Saúde no programa de pós-graduação em administração da Escola de Administração da Universidade Federal do Rio Grande do Sul (UFRGS).

\section{AGRADECIMENTO}

Agradecemos à direção administrativa do Instituto de Cardiologia, nas pessoas dos Srs. Rogério Pires e Aramy P. Silva, que permitiu à controladoria da instituição fornecer os dados que serviram de base para a realização deste trabalho.

\section{CONFLITO DE INTERESSES}

Os autores declararam inexistência de conflito de interesses relacionado a este manuscrito.

\section{REFERÊNCIAS}

1. Sacket DL, Straus SE, Richardson WS, Rosemberg W, Haynes RB. Medicina baseada em evidências: prática e ensino. 2a ed. Porto Alegre: Artmed; 2003.

2. Straus SE, Richardson WS, Glasziou P, Haynes RB. Evidencebased medicine: how to practice and teach EBM. $3^{\text {rd }}$ ed. London: Elsevier; 2005.

3. Porter ME, Teisberg EO. Repensando a saúde. Porto Alegre: Artmed; 2007.

4. Guandalini G, Borsato C. A inflação da saúde. Revista Veja, São Paulo. 2008;2060(19):95.

5. Padoveze CL. Planejamento orçamentário. São Paulo: Thomson; 2005.

6. Zdanowicz JE. Planejamento financeiro e orçamento. $2^{\text {a }}$ ed. Porto Alegre: Sagra-Luzzato; 1998.

7. Castello H. Gestão em hemodinâmica; 2008. [citado 17 ago. 2009]. Disponível em: http://www.sbhci.org.br.

8. Souza A. The incidence of DES USE in Latin America: can we do better? In: XII Congresso da SOLACI/XXVIII Congresso da SBHCl 2006, Porto Alegre.

9. DATASUS. Informações sobre hemodinâmica. [citado 17 ago. 2008]. Disponível em: http://www.datasus.org.

10. lanesko JA. Orçamento econômico-financeiro: uma contribuição relevante para a tomada de decisões nas empresas. UNOPAR científica. Ciências Jurídicas e Empresariais (Londrina). 2001; 2:119-40.

11. Tancredi FC, Barros SRL, Ferreira JHG. Planejamento em saúde. São Paulo: Instituto para Desenvolvimento da Saúde. Núcleo de Assistência Médico-Hospitalar; 1998.

12. Jacques JE. Gestão hospitalar: os custos médicos-assistenciais. São Leopoldo: UNISINOS; 2006.

13. Beulke R, Berto D. Gestão de custos e resultados na saúde. 4a ed. São Paulo: Saraiva; 2008. 